\title{
Type IV RTA in Chronic Adrenal Insufficiency and Concomitant Lisinopril Treatment
}

\author{
Francesca Galbiati \\ Department of Medicine, University of Pittsburgh Medical Center, 200 Lothrop Street, Suite N715, Pittsburgh, PA 15213, USA \\ Correspondence should be addressed to Francesca Galbiati; galbiatif@upmc.edu
}

Received 7 July 2020; Revised 30 September 2020; Accepted 9 October 2020; Published 19 October 2020

Academic Editor: Osamu Isozaki

Copyright (c) 2020 Francesca Galbiati. This is an open access article distributed under the Creative Commons Attribution License, which permits unrestricted use, distribution, and reproduction in any medium, provided the original work is properly cited.

\begin{abstract}
Type IV renal tubular acidosis (RTA) is the only RTA characterized by hyperkalemia, and it is caused by a true aldosterone deficiency or renal tubular aldosterone hyporesponsiveness. It is frequent among hospitalized patients as it is related to type 2 diabetes mellitus (T2DM) and common medications such as ACE-inhibitors (ACE-is) and trimethoprim-sulfamethoxazole (TMP-SMX). Drug-induced RTA commonly manifests in patients with predisposing conditions such as mild renal insufficiency and certain pharmacological therapies. ACE-i use and chronic adrenal insufficiency (cAI) are other significant risk factors. Chronic ACTH suppression is thought to induce global adrenal atrophy, including the zona glomerulosa, thus affecting aldosterone secretion as well. Furthermore, in the setting of cAI, treatment with ACE-is further suppresses aldosterone production. This case report describes a patient with cAI secondary to corticosteroid use for years who developed type IV RTA in the setting of lisinopril use. Potassium (K) elevation persisted despite removing underlying conditions and metabolic acidosis correction. The patient required long-term treatment with mineralocorticoids in addition to sodium bicarbonate to maintain normal $\mathrm{K}$ levels and acid-base status. Mineralocorticoid administration is a second-line treatment for type IV RTA, but it might be necessary for a subgroup of high-risk patients. In fact, it is important to consider patients with chronic adrenal insufficiency and on ACE-is treatment at increased risk for refractory hyperkalemia in the setting of type IV RTA. Indeed, this subgroup of patients can have severe hypoaldosteronism.
\end{abstract}

\section{Introduction}

Type IV renal tubular acidosis (RTA) is the only RTA characterized by hyperkalemia, and it is caused by a true aldosterone deficiency or renal tubular aldosterone hyporesponsiveness [1]. It is frequent among hospitalized patients as it is related to type 2 diabetes mellitus (T2DM) and common medications such as ACE-inhibitors (ACEis) and trimethoprim-sulfamethoxazole (TMP-SMX), and it occurs more often in patients with chronic kidney disease (CKD) [2]. Drug-induced RTA commonly manifests in patients with predisposing conditions, for example, the use of diclofenac and mild renal insufficiency exacerbated the potassium-altering effect of trimethoprim [3]. ACE-i use ${ }^{\circ}$ and chronic adrenal insufficiency (cAI) are other significant risk factors. It is well known that chronic steroid treatment is a major cause of ACTH suppression [4]. Chronic ACTH suppression is thought to induce global adrenal atrophy $[4,5]$, including the zona glomerulosa, thus affecting aldosterone secretion as well. In cAI, treatment with ACE-is further suppresses aldosterone production by inhibiting the angiotensin-converting enzyme (Figure 1). In the clinical setting of $\mathrm{cAI}$ and concomitant ACE-i treatment, type IV RTA can be refractory to first-line interventions with subsequent persistent hyperkalemia requiring treatment with mineralocorticoids in addition to sodium bicarbonate. The literature on type IV RTA in patients with secondary or iatrogenic causes of hypoaldosteronism is sparse and requires more data. 


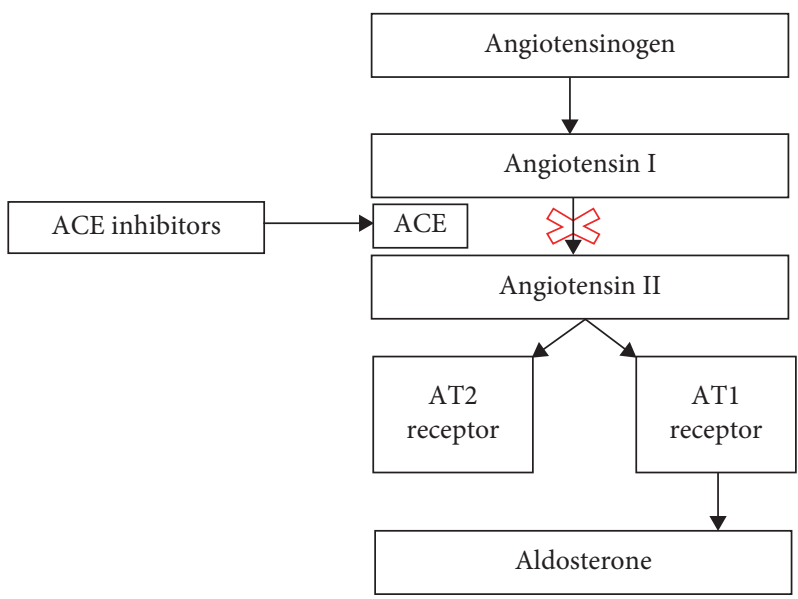

FIgURE 1: Effects of ACE-inhibitors on angiotensin conversion. ACE-inhibitors suppress the ACE enzyme action, thus blocking the conversion of angiotensin I to angiotensin II and its action on AT2 and AT1 receptors. The interaction between angiotensin II and AT1 receptors leads to aldosterone production.

\section{Case Presentation}

A 66-year-old female with a history of rheumatoid arthritis on prednisone $5 \mathrm{mg}$ daily, T2DM, CKD, AI secondary to chronic steroid use, and recent hospitalization for methicillin-resistant Staphylococcus aureus (MRSA) osteomyelitis still on antibiotics, now admitted for cholangitis treatment, suddenly presents unexplained hyperkalemia. At the time of admission, the patient was started on piperacillin-tazobactam for cholangitis and on TMP-SFX for a history of MRSA osteomyelitis. She also received stress-dose steroids for 48 hours with immediate clinical improvement. The patient's hospitalization was prolonged (more than 30 days) and complicated by acute kidney injury, hyperkalemia, and nonanion gap metabolic acidosis (NAGMA) outlined as follows. The patient also had a history of hypertension treated with lisinopril $5 \mathrm{mg}$ daily, which was initially discontinued and restarted during the hospitalization (day 30) at the increased dose of $10 \mathrm{mg}$ daily given severe hypertension. On day 33, the patient's creatinine rose to $1.5 \mathrm{mg} / \mathrm{dL}$ from a baseline of $1.2 \mathrm{mg} / \mathrm{dL}$, and her previously normal potassium (K) increased to $5.5 \mathrm{mmol} / \mathrm{L}$ (Table 1). This was thought to be related to lisinopril, which was discontinued. Off lisinopril, her kidney function recovered, but unexpectedly, hyperkalemia worsened to $6.3 \mathrm{mmol} / \mathrm{L}$, and she presented a new-onset NAGMA with serum bicarbonate $17.5 \mathrm{mmol} / \mathrm{L}$. The abnormally elevated $\mathrm{K}$ was then attributed to TMP-SMX-induced hyperkalemia, so TMP-SFX was switched to doxycycline, and the patient was given patiromer. Despite these interventions, both hyperkalemia and NAGMA persisted $(\mathrm{K}, 6.3 \mathrm{mmol} / \mathrm{L}$ and bicarbonate, $19.6 \mathrm{mmol} / \mathrm{L}$ on day 35$)$. At that point, urine electrolytes and venous blood gas were obtained, demonstrating a positive urine anion gap and venous $\mathrm{pH}$ of 7.31 . The urinalysis was normal with $\mathrm{pH}$ of 7 , no evidence of infection, and absence of proteinuria, glycosuria, and microhematuria. Plasma renin activity was 0.95 , and aldosterone levels were undetectable; however, the treatment with ACE-I could affect the interpretation of this test. The picture was now consistent with type IV RTA, so the patient was started on oral sodium bicarbonate $1300 \mathrm{mg}$ three times a day with the resolution of NAGMA. Despite the discontinuation of possible culprit medications, the patient was still hyperkalemic. Finally, considering her cAI with a likely component of hypoaldosteronism worsened by lisinopril, fludrocortisone $0.1 \mathrm{mg}$ was added daily with immediate normalization of $\mathrm{K}$ (from $5.2 \mathrm{mmol} / \mathrm{L}$ to $4.4 \mathrm{mmol} / \mathrm{L}$ ). This happened the fourth day after stopping lisinopril (Table 1). The patient had to continue the daily therapy with both sodium bicarbonate and fludrocortisone to maintain normal $\mathrm{K}$ levels and acid-base status and did not experience any electrolyte imbalance or worsening hypertension, while on the mineralocorticoid replacement therapy, as a demonstration that, she was indeed aldosterone-deficient. Of note, cortisol and adrenocorticotropic hormone levels were not measured during this admission as the patient's diagnosis of adrenal insufficiency was well established, and she was on treatment.

\section{Discussion}

The classical manifestations of type IV RTA are hyperkalemia and NAGMA. In the inpatient setting, these are frequently primarily attributed to patients' comorbidities and polypharmacy, thus missing the correct diagnosis. The type IV RTA workup (serum K, urine electrolytes and anion gap, urine $\mathrm{pH}$, and urine bicarbonate) is cost-effective and straightforward and should be included in first-line tests for hyperkalemia workup. It is, in fact, crucial to address diagnosis and treatment quickly, given the risk of hyperkalemia in hospitalized patients [6]. However, healthcare providers often prescribe expensive tests and medications (i.e., newer potassium binders) to address hyperkalemia with delays in the diagnosis and increase in healthcare expenses.

When approaching patients with cAI and on ACE-is presenting with hyperkalemia, it is important to consider a primary adrenal insufficiency-like picture. The chronic ACTH suppression by exogenous steroid medications can cause adrenal atrophy and subphysiological aldosterone 
TABLE 1: Hospital course timeline starting from day 30; medication management; serum potassium, bicarbonate, and creatinine levels.

\begin{tabular}{|c|c|c|c|}
\hline Day of admission & $\begin{array}{c}\text { Potassium } \\
(\mathrm{mmol} / \mathrm{L})\end{array}$ & $\begin{array}{l}\text { Bicarbonate } \\
(\mathrm{mmol} / \mathrm{L})\end{array}$ & $\begin{array}{l}\text { Creatinine } \\
(\mathrm{mg} / \mathrm{dL})\end{array}$ \\
\hline 30 & 4.0 & 21.6 & 1.2 \\
\hline \multicolumn{4}{|c|}{ Lisinopril $10 \mathrm{mg}$ daily was restarted on day 30} \\
\hline 31 & 3.6 & 21.9 & 1.3 \\
\hline 32 & 3.9 & 21.3 & 1.4 \\
\hline 33 & 5.5 & 17.5 & 1.5 \\
\hline \multicolumn{4}{|c|}{ Lisinopril discontinued due to acute kidney injury on day 33} \\
\hline 34 & 5.9 & 19.1 & 1.7 \\
\hline \multicolumn{4}{|c|}{$\begin{array}{l}\text { TMP-SMX was switched to doxycycline; the patient received patiromer } \\
\text { on day } 34\end{array}$} \\
\hline 35 & 6 & 19.6 & 1.4 \\
\hline 35 & 6.3 & 19.6 & 1.5 \\
\hline \multicolumn{4}{|c|}{ Sodium bicarbonate $1300 \mathrm{mg}$ three times a day was started on day 35} \\
\hline 36 & 5.5 & 20.5 & 1.2 \\
\hline \multicolumn{4}{|c|}{ Fludrocortisone $0.1 \mathrm{mg}$ daily was started on day 36} \\
\hline 37 & 4.4 & 20.8 & 1.1 \\
\hline 38 & 4.5 & 21.6 & 1.2 \\
\hline Discharge & & & \\
\hline
\end{tabular}

Values of serum potassium, serum bicarbonate, and serum creatinine levels over the course of admission and treatment modifications secondary to acute kidney injury, hyperkalemia, and NAGMA. Based on our laboratory normal range, abnormal values are highlighted in bold.

levels in addition to hypocortisolemia. The additive inhibition of the angiotensin-converting enzyme by ACE-is further affects aldosterone production, thus inducing significant hypoaldosteronism.

The limitations of this case report are that, with only one patient, we cannot formulate robust evidence-based guidance on the treatment of type IV RTA in the particular condition of concomitant CAI and ACE-is use. Also, we do not have a documented measure of cortisol and ACTH in our patient at the time of presentation. Last, a combination of medications, such as bactrim and lisinopril, and cAI can explain type IV RTA in this patient; however, the prompt response to fludrocortisone and the absence of side effects on a long-term mineralocorticoid replacement therapy is indicative of a true aldosteronism deficiency.

\section{Conclusion}

In patients with unexplained hyperkalemia and underlying predisposing conditions, type IV RTA must always be considered. In particular, the index of suspicion must be high in patients with cAI and on concomitant ACE-I treatment leading to further suppression of aldosterone activity. Occasionally, in secondary or iatrogenic hypoaldosteronism, refractory hyperkalemia responds only to sodium bicarbonate and fludrocortisone given in combination.

\section{Abbreviations}
RTA: $\quad$ Renal tubular acidosis
T2DM: $\quad$ Type 2 diabetes mellitus
ACE-is: Angiotensin-converting enzyme inhibitors
TMP-SMX: Trimethoprim sulfamethoxazole
CKD: $\quad$ Chronic kidney disease
cAI: Chronic adrenal insufficiency
MRSA: Methicillin-resistant Staphylococcus aureus

NAGMA: Nonanion gap metabolic acidosis

K: Potassium.

\section{Data Availability}

No data were used to support this study as this is a case report on one patient and information is confidential. Additional data can be made available from the corresponding author upon request.

\section{Conflicts of Interest}

The authors declare that they have no conflicts of interest.

\section{References}

[1] F. E. Karet, "Mechanisms in hyperkalemic renal tubular acidosis: figure 1," Journal of the American Society of Nephrology, vol. 20, no. 2, pp. 251-254, 2009.

[2] D. Batlle and J. Arruda, "Hyperkalemic forms of renal tubular acidosis: clinical and pathophysiological aspects," Advances in Chronic Kidney Disease, vol. 25, no. 4, pp. 321-333, 2018.

[3] M. A. Perazella, "Trimethoprim-induced hyperkalaemia," Drug Safety, vol. 22, no. 3, pp. 227-236, 2000.

[4] A. K. Younes and N. K. Younes, "Recovery of steroid induced adrenal insufficiency," Translational Pediatrics, vol. 6, no. 4, pp. 269-273, 2017.

[5] N. El Ghorayeb, I. Bourdeau, and A. Lacroix, "Role of ACTH and other hormones in the regulation of aldosterone production in primary aldosteronism," Frontiers in Endocrinology, vol. 7, no. 72, 2016.

[6] M. S. Stevens and R. W. Dunlay, "Hyperkalemia in hospitalized patients," International Urology and Nephrology, vol. 32, no. 2, pp. 177-180, 2000. 